\title{
Finsler Tractography for White Matter Connectivity Analysis of the Cingulum Bundle
}

\author{
John Melonakos ${ }^{1}$, Vandana Mohan ${ }^{1}$, Marc Niethammer ${ }^{2}$, Kate Smith ${ }^{2}$, \\ Marek Kubicki², and Allen Tannenbaum ${ }^{1}$ \\ ${ }^{1}$ Department of Electrical and Computer Engineering, Georgia Institute of \\ Technology, USA \\ jmelonak@ece.gatech.edu \\ ${ }^{2}$ Psychiatry Neuroimaging Laboratory, Harvard Medical School, USA
}

\begin{abstract}
In this paper, we present a novel approach for the segmentation of white matter tracts based on Finsler active contours. This technique provides an optimal measure of connectivity, explicitly segments the connecting fiber bundle, and is equipped with a metric which is able to utilize the directional information of high angular resolution data. We demonstrate the effectiveness of the algorithm for segmenting the cingulum bundle.
\end{abstract}

\section{Introduction}

Since the advent of diffusion weighted magnetic resonance imaging (DW-MRI), a great amount of research has been devoted to finding and characterizing neural connections between brain structures. In this paper, we present a novel approach for the segmentation of white matter tracts based on Finsler active contours [1]. Furthermore, we show results of the algorithm for segmenting the cingulum bundle (CB).

Recently, tractography advances have been made which provide full brain optimal connectivity maps from predefined seed regions. These approaches can be subdivided into stochastic and energy-minimization approaches.

Stochastic approaches produce probability maps of connectivity between a seed region and the rest of the brain. Parker et al. developed PICo, a probabilistic index for standard streamline techniques [2. Perrin et al. presented probabilistic techniques for untangling fiber crossings using q-ball fields 3 . In other work, Friman et al. proposed a method for probabilistically growing fibers in a large number of random directions and inferring connectivity from the resulting percentages of connections between seed and target regions [4. While providing

\footnotetext{
* This work was supported in part by grants from NSF, AFOSR, ARO, MURI, MRIHEL as well as by a grant from NIH (NAC P41 RR-13218) through Brigham and Women's Hospital. This work is part of the National Alliance for Medical Image Computing (NAMIC), funded by the National Institutes of Health through the NIH Roadmap for Medical Research, Grant U54 EB005149. Information on the National Centers for Biomedical Computing can be obtained from http://nihroadmap.nih.gov/bioinformatics.
} 
a measure of connectivity between brain regions, these stochastic approaches do not provide an explicit segmentation of the fiber bundle itself.

Energy-minimization techniques have also been developed. Parker et al. proposed fast marching tractography which minimizes an energy based on both the position and direction of the normal to a propagating front [5]. O'Donnell et al. cast the tractography problem in a geometric framework finding geodesics on a Riemannian manifold based on diffusion tensors 6]. Similarly, Prados et al. and Lenglet et al. demonstrated a Riemannian based technique, GCM (Geodesic Connectivity Mapping), for computing geodesics using a variant of fast marching methods adapted for directional flows [78. Jackowski et al. also find Riemannian geodesics using Fast Sweeping methods as given by Kao et al. 91011. In cases of high angular diffusion data, these Riemannian based approaches do not take advantage of the full directional resolution due to the loss of information incurred by the construction of diffusion tensors.

In this paper, we present a technique which provides an optimal measure of connectivity, explicitly segments the connecting fiber bundle, and is based on the richer Finsler metric. Rather than following the traditional approach of finding a large number of fibers (which individually have questionable meaning), clustering them, and then performing statistical analyses on the clusters, we present an alternative approach. We first find the optimal connection, which we term the anchor tract, on the Finsler manifold between the seed and target regions. Then, we initialize an expanding surface level set evolution on the anchor tract which grows until it stops at a local minima on the edge of the fiber bundle. Finally, since the fiber bundle extraction does not rely upon standard statistical measures, such as fractional anisotropy (FA), for the segmentation, we are free to use these measures to statistically compare fiber bundles in clinical studies.

In Section 2, we motivate our interest in segmenting the cingulum bundle. In Section 3, we describe the algorithm for extracting the anchor tracts. Then, in Section 4, we present our surface evolution algorithm for extracting the full cingulum bundle. Finally, in Section 5 , we show results for extracting the anchor tracts and the corresponding cingulum bundle.

\section{The Cingulum Bundle}

The cingulum bundle is a $5-7 \mathrm{~mm}$ in diameter fiber bundle that interconnects all parts of the limbic system. It originates within the white matter of the temporal pole, and runs posterior and superior into the parietal lobe, then turns, forming a "ring-like belt" around the corpus callosum, into the frontal lobe, terminating anterior and inferior to the genu of the corpus callosum in the orbital-frontal cortex [12. Because of its involvement in executive control and emotional processing, the cingulum bundle has been investigated in several clinical populations, including depression and schizophrenia. Previous studies, using DW-MRI, in schizophrenia, demonstrated decrease of FA in anterior part of the cingulum bundle [1314, at the same time pointing to the technical limitations restricting these investigations from following the entire fiber tract. 


\section{Anchor Tracts on a Finsler Manifold}

In this section, we present our algorithm for extracting the optimal path, or anchor tract, between two regions in the brain. In this formulation, the optimal path is defined with respect to a Finsler metric. In the case of data acquired with only 6 gradient directions, this reduces to a Riemannian metric because there are 6 independent elements of the diffusion tensor. However, in the case of high angular data, the Finsler metric is more flexible than the Riemannian metric as it is not restricted to an ellipsoidal diffusion profile which results from the Gaussian diffusion assumption.

In order to find the anchor tract, we construct a dynamic programming based approach which uses a Fast Sweeping method, see [15]1011] for algorithmic details. Also, see the work by Jackowski et al. for a formulation of this algorithm based on the Riemannian metric 9]. For the sake of completeness, we include a brief overview of the algorithm.

For any given starting point $\boldsymbol{p}_{0}$, define the value function as the minimum cost for reaching a seed region $S \subset \mathbb{R}^{n}$ from $\boldsymbol{p}_{0}$. The resulting Hamilton-JacobiBellman equation is

$$
\left\{\begin{array}{l}
0=\inf _{\hat{\boldsymbol{d}} \in S^{n-1}}\left\{\psi(\boldsymbol{p}, \hat{\boldsymbol{d}})+\nabla \mathcal{L}^{*}(\boldsymbol{p}) \cdot \hat{\boldsymbol{d}}\right\} \\
\mathcal{L}^{*}(\boldsymbol{s})=0 \text { for } \boldsymbol{s} \in S
\end{array}\right.
$$

where $\psi$ is the local cost at each point, $\boldsymbol{p}$, and for each direction, $\hat{\boldsymbol{d}}$, and $\mathcal{L}^{*}$ is the optimal Finsler length. Numerically, this equation may be solved via Fast Sweeping as shown in Algorithm 1 .

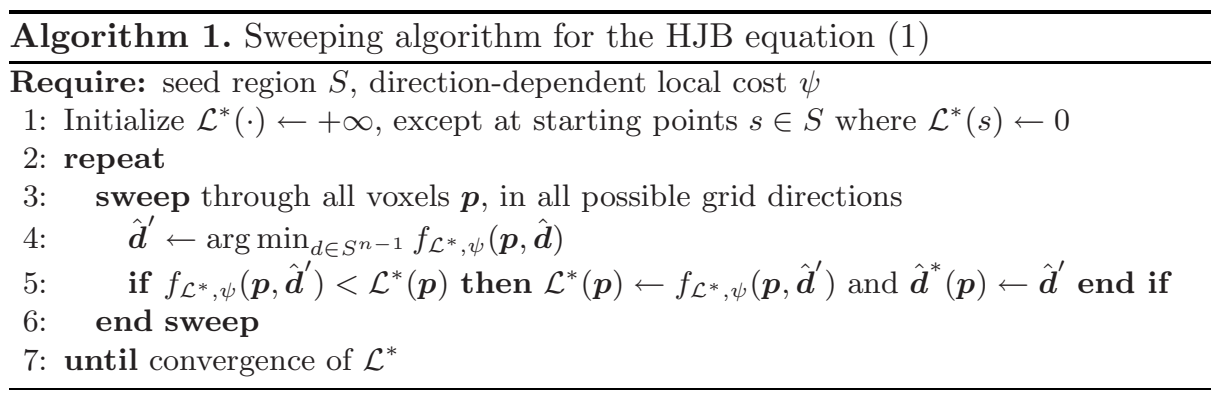

The Fast Sweeping algorithm results in optimal connectivity maps and characteristic vectors at every point in the domain. The anchor tract is then determined by following the characteristic vectors from the target region back to the seed region, not by gradient descent as is standard in direction-independent schemes [16].

There are many numerical schemes which may be used to solve the HamiltonJacobi-Bellman equation given above. The number of sweeping iterations required by the Fast Sweeping algorithm depends upon the number of turns in the 
optimal path. Since neural tracts tend to have few total turns, the Fast Sweeping algorithm is efficient for extracting tracts. We also note that the connections of graph cuts and such directional metrics have been described in [17/8]19]. Of particular note, in [18] the explicit connection between Finsler distances and the flux methods of [20] is considered in some detail.

\section{Level Set Fiber Bundle Segmentation}

In this section, we discuss the level set surface evolution which we have used to extract the volumetric cingulum bundle. This level set surface is initialized on the anchor tract described in Section 3 By using the calculus of variations, the minimizing flow for a directional cost $\psi(\boldsymbol{p}, \hat{\boldsymbol{d}})$ is obtained as,

$$
\Sigma_{t}=-\left\{\nabla_{\boldsymbol{p}} \psi \cdot N+\operatorname{Tr}\left(\nabla_{\hat{\boldsymbol{d}} \hat{\boldsymbol{d}}} \psi\right)+(n-1) \psi H\right\} N
$$

where $\Sigma$ is the evolving hypersurface, $N$ is the outward unit normal to the hypersurface, and $H$ denotes the mean curvature. A derivation of this flow can be found in [21]. The expression for the evolution of the level set function ' $u$ ' is obtained as,

$$
u_{t}=\left\{\nabla_{\boldsymbol{p}} \psi \cdot \nabla u\right\}+\left\{\operatorname{Tr}\left(\nabla_{\hat{\boldsymbol{d}} \hat{\boldsymbol{d}}} \psi\right)+(n-1) \psi H\right\}\|\nabla u\| .
$$

We use the sparse field method of Whitaker et al. to efficiently implement this level set surface evolution 22. We also use the angular interpolation algorithm presented by Tao et al. 23 .

In order to find the edge of the cingulum bundle, we construct a cost, $\psi$, which aligns the tangent plane of the hypersurface with edges in the directions of diffusion of the image volume. To produce a measure of these diffusion edges, we compute the positional gradient of each diffusion direction. We then construct $\psi$ as a function of these positional gradients.

Mathematically the form of the cost function that we desire to extremize can be written as follows.

$$
\psi(\boldsymbol{p}, \hat{\boldsymbol{d}})=\psi(\boldsymbol{p}, N(\boldsymbol{p}))=f\left(\nabla_{\boldsymbol{p}} \phi\left(\boldsymbol{p}, d_{1}\right) \cdot N, \nabla_{\boldsymbol{p}} \phi\left(\boldsymbol{p}, d_{2}\right) \cdot N \cdots \nabla_{\boldsymbol{p}} \phi\left(\boldsymbol{p}, \hat{\boldsymbol{d}}_{n_{s}}\right) \cdot N\right)
$$

where $d_{1}$ through $d_{n_{s}}$ denote the $n_{s}$ diffusion directions, $\phi$ denotes the DWI data, $N(\boldsymbol{p})$ denotes the normal to the hypersurface at position $\boldsymbol{p}$, and $f$ is a linear function of the arguments.

There are many possible choices for the function $\psi$. In this paper, we choose $\psi$ to be the following:

$$
\psi=\left\{\frac{1}{n_{s}} \cdot \sum_{i=0}^{n_{s}} \frac{\nabla_{\boldsymbol{p}} \phi\left(\boldsymbol{p}, d_{i}\right) \cdot N(\boldsymbol{p})}{\left\|\nabla_{\boldsymbol{p}} \phi\left(\boldsymbol{p}, d_{i}\right)\right\|}\right\}-1 .
$$

This cost ranges from $[-1,0]$ and is maximized (at 0) when the normal to the hypersphere is aligned with the mean gradient direction. 


\section{$5 \quad$ Experiments and Results}

In this section, we present segmentation results for anchor tracts of the right and left cingulum bundles of 12 schizophrenic and 12 normal patients. Further, we present a first implementation of the level set surface flow introduced in Section 4 . Scans were acquired on a 3 Tesla GE system (General Electric Medical Systems, Milwaukee, WI). We acquired 51 directions with $b=700 \frac{\mathrm{s}}{\mathrm{mm}^{2}}, 8$ baseline scans with $b=0 \frac{\mathrm{s}}{\mathrm{mm}^{2}}$. The following scan parameters were used: TR $17000 \mathrm{~ms}$, TE 78

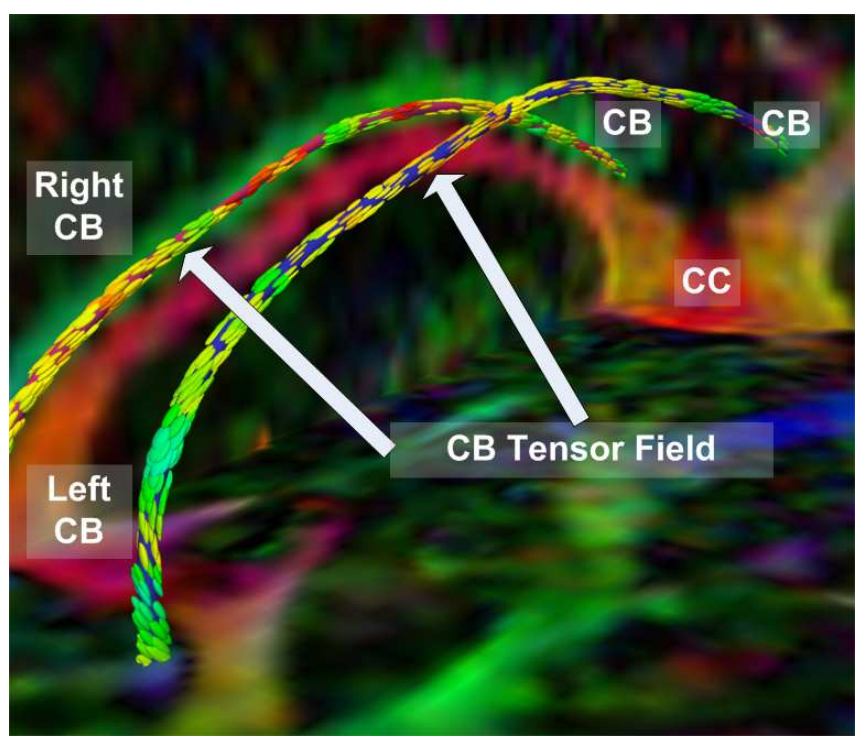

(a)

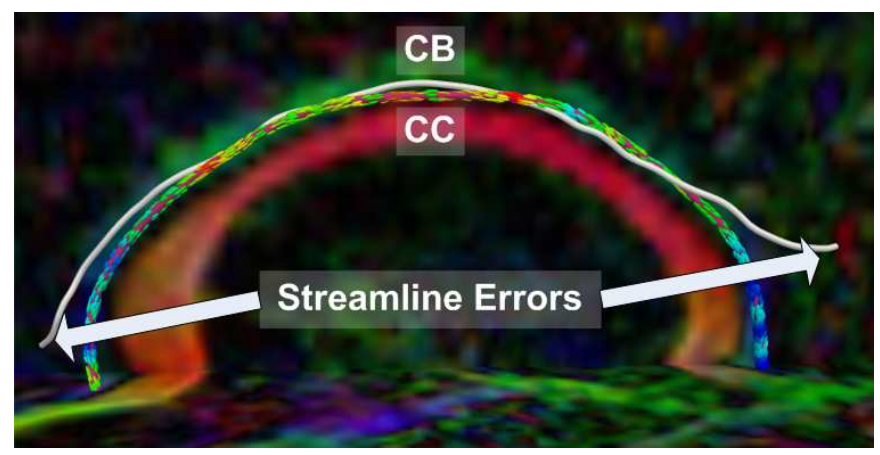

(b)

Fig. 1. Cingulum Bundle Anchor Tracts from: (a) detailed view of a normal control case, (b) streamline example on a schizophrenic case 


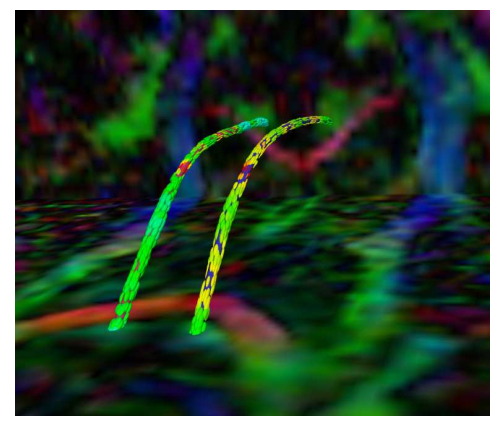

(a)

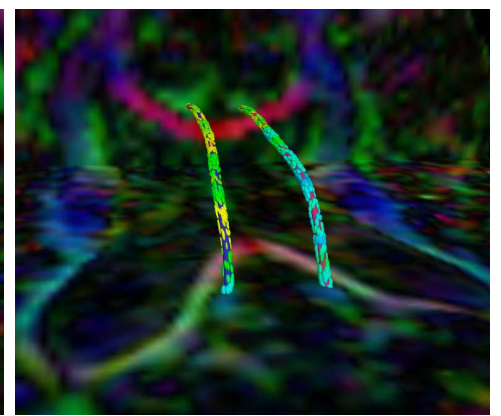

(b)

Fig. 2. Cingulum Bundle Anchor Tracts: (a) anterior and (b) posterior views of a normal control case

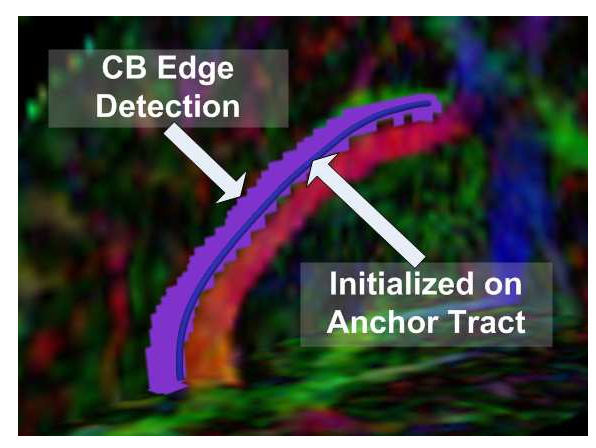

Fig. 3. Cingulum bundle level set segmentation result on a schizophrenic case

ms, FOV $24 \mathrm{~cm}, 144$ x 144 encoding steps, $1.7 \mathrm{~mm}$ slice thickness. We acquired 81 axial-oblique slices parallel to the AC-PC line covering the whole brain.

Manually selected endpoints were provided by experts for each case. Masking is necessary to ensure that the tract does not take shortcuts through cerebral spinal fluid (CSF). We used a simple thresholding on the trace of the tensors to mask out CSF regions. Furthermore, we applied a threshold on strong left-right diffusion to mask out the corpus callosum which runs inferior to the cingulum bundle.

First, we show results of extracting the cingulum bundle anchor tract. In each figure, the tracts are superimposed upon the diffusion data which has been colored by direction (e.g. green signifies high anterior-posterior diffusion). The tensors, shown for convenience, are colored by FA. In Fig. 11 we depict the advantage of this algorithm in segmenting the cingulum bundle. The cingulum bundle curves around the ventricles on both the anterior and posterior ends. In Fig. 1(a) we show how the anchor tracts follow the smoothly varying tensor field around the bend of the ventricles. In Fig. 1(b), we share an example of a streamline based approach (freely available in the 3D Slicer tool) which fails 
in the same bend around the ventricles. This is the area where large tracts are joining the main bundle from both medial and lateral parts of the parietal lobes. Using the full directional data, our method is able to resolve the cingulum bundle in the presence of the merging fibers. In Fig. 2. we show anterior and posterior zoomed-out views of the anchor tracts.

Next, we show the result of an edge-based surface level set evolution on a portion of the cingulum bundle. We present here a proof of concept implementation of the theory from Section 4 . In our experiment, the level set flow converged to the edge of the bundle as depicted in the image.

\section{Conclusions and Future Work}

In this work, we have introduced a novel approach for the segmentation of white matter tracts. We have shown an application of the method for the segmentation of the cingulum bundle. Given manually selected endpoints, the algorithm automatically extracts the cingulum bundle.

We have shown how the method was able to find centerlines, or anchor tracts of the cingulum bundle on 24 cases. We have also shown a first implementation of the edge-based level set surface extraction. In the future, we will run the edge-based level set segmentation on all 24 cases. We will also explore the use of other cost functions, especially region-based costs, for the level set segmentation. Finally, we will be able to compute statistics across the resulting segmented cingulum bundles for the population of schizophrenics and normal controls.

\section{References}

1. Melonakos, J., Pichon, E., Angenent, S., Tannenbaum, A.: Finsler active contours. IEEE Transactions on Pattern Analysis and Machine Intelligence, Preprint available online (2007)

2. Parker, G., Haroon, H., Wheeler-Kingshott, C.: A framework for a streamline-based probabilistic index of connectivity(PICo) using a structural interpretation of MRI diffusion measurements. Journal of Magnetic Resonance Imaging 18(2), 242-254 (2003)

3. Perrin, M., Poupon, C., Cointepas, Y., Rieul, B., Golestani, N., Pallier, C., Riviere, D., Constantinesco, A., Le Bihan, D.: Fiber tracking in Q-ball fields using regularized particle trajectories. Proc. of IPMI 2(3) (2005)

4. Friman, O., Farnebäck, G., Westin, C.: A Bayesian approach for stochastic white matter tractography. IEEE Transactions on Medical Imaging 25(8), 965 (2006)

5. Parker, G., Wheeler-Kingshott, C., Barker, G.: Estimating distributed anatomical connectivity using fast marchingmethods and diffusion tensor imaging. Medical Imaging, IEEE Transactions 21(5), 505-512 (2002)

6. O'Donnell, L., Haker, S., Westin, C.: New Approaches to Estimation of White Matter Connectivity in Diffusion Tensor MRI: Elliptic PDEs and Geodesics in a Tensor-Warped Space. In: Dohi, T., Kikinis, R. (eds.) MICCAI 2002. LNCS, vol. 2488, pp. 459-466. Springer, Heidelberg (2002) 
7. Prados, E., Lenglet, C., Pons, J., Wotawa, N., Deriche, R., Faugeras, O., Soatto, S.: Control Theory and Fast Marching Techniques for Brain Connectivity Mapping. In: Proceedings of the 2006 IEEE Computer Society Conference on Computer Vision and Pattern Recognition, vol. 1, pp. 1076-1083 (2006)

8. Lenglet, C., Rousson, M., Deriche, R., Faugeras, O., Lehericy, S., Ugurbil, K.: A Riemannian Approach to Diffusion Tensor Images Segmentation. In: Christensen, G.E., Sonka, M. (eds.) IPMI 2005. LNCS, vol. 3565, pp. 591-602. Springer, Heidelberg (2005)

9. Jackowski, M., Kao, C., Qiu, M., Constable, R., Staib, L.: White matter tractography by anisotropic wavefront evolution and diffusion tensors imaging. Medical Image Analysis 9, 427-440 (2005)

10. Kao, C., Osher, S., Qian, J.: Lax-Friedrichs sweeping scheme for static HamiltonJacobi equations. Journal of Computational Physics 196(1), 367-391 (2004)

11. Kao, C., Osher, S., Tsai, Y.: Fast sweeping methods for static Hamilton-Jacobi equations. SIAM journal on numerical analysis 42(6), 2612-2632 (2005)

12. Schmahmann, J., Pandya, D.: Fiber Pathways of the Brain. Oxford University Press, Oxford (2006)

13. Kubicki, M., Westin, C., Nestor, P., Wible, C., Frumin, M., Maier, S., Kikinis, R., Jolesz, F., McCarley, R., Shenton, M.: Cingulate fasciculus integrity disruption in schizophrenia: a magnetic resonance diffusion tensor imaging study. Biological Psychiatry 54(11), 1171-1180 (2003)

14. Wang, F., Sun, Z., Cui, L.: Du, X., Wang, X., Zhang, H., Cong, Z., Hong, N., Zhang, D.: Anterior Cingulum Abnormalities in Male Patients With Schizophrenia Determined Through Diffusion Tensor Imaging (2004)

15. Pichon, E., Westin, C., Tannenbaum, A.: A Hamilton-Jacobi-Bellman approach to high angular resolution diffusion tractography. In: Duncan, J.S., Gerig, G. (eds.) MICCAI 2005. LNCS, vol. 3749, pp. 180-187. Springer, Heidelberg (2005)

16. Lin, Q.: Enhancement, extraction, and visualization of 3D volume data. Department of Electrical Engineering, Linköping University (2003)

17. Boykov, Y., Kolmorgorov, V., Cremers, D., Delong, A.: An integral solution to surface evolution PDEs via geo-cuts. In: Proceedings IEEE European Conference Computer Vision, pp. 409-422. IEEE Computer Society Press, Los Alamitos (2006)

18. Kolmorgorov, V., Boykov, Y.: What metrics can be approximated by geo-cuts or global optimization of length/area and flux. In: Proceedings IEEE International Conference Computer Vision, IEEE Computer Society Press, Los Alamitos (2003)

19. Boykov, Y., Kolmorgorov, V.: Computing geodesics and minimal surfaces via graph cuts. In: Proceedings IEEE International Conference Computer Vision, pp. 26-33. IEEE Computer Society Press, Los Alamitos (2003)

20. Vasilevsky, A., Siddiqi, K.: Flux maximizing geometric flows. IEEE PAMI 24, 15651579 (2002)

21. Pichon, E.: Novel methods for multidimensional image segmentation. PhD thesis, Georgia Institute of Technology (2005)

22. Whitaker, R.: A Level-Set Approach to 3D Reconstruction from Range Data. International Journal of Computer Vision 29(3), 203-231 (1998)

23. Tao, X., Miller, J.V.: A method for registering diffusion weighted magnetic resonance images. In: Larsen, R., Nielsen, M., Sporring, J. (eds.) MICCAI 2006. LNCS, vol. 4191, pp. 594-602. Springer, Heidelberg (2006) 